\title{
Relative preferences for various bivalued ratio schedules
}

\author{
WILLIAM AHEARN and PHILIP N. HINELINE \\ Temple University, Philadelphia, Pennsylvania \\ and \\ F. G. DAVID \\ University of The Philippines, Quezon City, The Philippines
}

\begin{abstract}
Widely cited experiments on optimal foraging have used bivalued distributions as representing environmental stochasticity, characterizing these in terms of their arithmetic means. In contrast, research on free-operant choice has established that organisms prefer variable patterns of food delivery, relative to fixed patterns with the same mean values. To explore such departures from linear averaging, specifically with respect to bivalued alternatives, pigeons were given choices between a fixed-ratio (FR) schedule of food delivery that required 15, 30, or 60 responses and bivalued variable-ratio schedules with an arithmetic mean of 60.5 or 60 . A bivalued schedule of 1 and 120 was preferred almost exclusively over each of the FR values. With a bivalued schedule of 15 and 105, there was a shift of preference, most notably in the FR-15 condition, but in no case was linear averaging a good predictor of the birds' choices. Geometric averaging fared better, but even this failed to represent the apparent salience of the minimum value of the bivalued schedule in some conditions.
\end{abstract}

The present research addresses an intersecting concern of theorists who work in the biological tradition of behavioral ecology and those who work in the psychological tradition of behavior analysis: animals' choices in situations of uncertainty. Behavioral ecologists have framed the relevant issues in relation to theories of optimal foraging, especially models that have addressed environmental stochasticity and sensitivity to risk. In the words of Caraco, Martindale, and Whittam (1980), “Birds' foraging preferences for variable rewards respond not only to the mean, but also the variance of food rewards. ... We believe that foraging models should consider environmental stochasticity and an animal's response to this variation" (p. 820). Theories of foraging have expressed stochasticity in terms of distributions of varied outcomes, usually summarized by the arithmetic mean as "expected value."

Related experimentation, however, often has failed to address whether, and in what ways, the experimental subjects are sensitive to different characteristics of variability, or even whether the mean is an appropriate summary statistic. For example, in the experiments by Caraco et al. (1980) that inspired the above quotation, indifference curves were constructed by giving birds choices between fixed and bivalued distributions in amount of food, with

This research was supported in part by a Grant-In-Aid of Research from Temple University to the second author. Participation of the third author was supported by NSF Grant 9015810 to Temple University under the program for Science in Developing Countries. Reprints can be obtained from the first or second author at the Department of Psychology, Weiss Hall, Temple University, Philadelphia, PA 19122. probability counterbalanced against amount such that overall food availability was relatively constant across alternatives. The birds' preferences were then characterized in terms of "risk proneness" or "risk aversion." There are flawed assumptions in that counterbalancing, for Leventhal, Morrell, Morgan, and Perkins (1959) found, when examining rats' behavior in E-shaped alley mazes, that probability and amount of food do not compose a linear tradeoff with respect to reinforcement effects. Furthermore, as Mazur (1988) has shown, manipulations (such as food deprivation) that were interpreted as affecting tolerance for risk may have affected preference for larger food items irrespective of risk. These problems can be prevented by comparing fixed and variably distributed food items of constant size, thus eliminating the confound between size and probability. But even with this precaution, the bivalued distribution is a tenuous representative of the environmental stochasticity that foraging theories claim to address. Its statistical expected value implies central tendency where there is none, and it has no smooth envelope such as the term "distribution" suggests. Whatever kinds of distributions are to be used, the subjects' sensitivities to particular distributional characteristics need to be assessed.

The present experiments were designed to be relevant to work in the foraging tradition while examining subjects' repeated choices between fixed and probabilistic outcomes in a laboratory situation. Thus, we have capitalized upon classic work on operant schedules of reinforcement, where size of food items is typically held constant while other characteristics of their availability are systematically varied. That work has had some of its 
own blind spots, however, with respect to identifying specific characteristics of variability that affect preference. In the early years of operant research, variable-interval (VI) and variable-ratio (VR) schedules were extensively studied in experiments where the specific characteristics of the variability were seldom specified. One of the first to remedy this deficit was Killeen (1968), who examined pigeons' choices between fixed-interval (FI) and three different VI schedules (one based on arithmetic and two based on geometric progressions of intervals). By adjusting the FI duration in each case to identify an indifference point relative to each VI schedule, Killeen found that preferences were proportional to relative rates of reinforcement computed in terms of harmonic means. In a second experiment, Killeen studied the birds' choices between two VI schedules, one of which was an arithmetic progression (VI-40), the other being a geometric progression (VI-80). The harmonic mean interval of the VI-40 (24.6) was greater than that of the VI-80 (11.5), while the arithmetic mean of the VI-40 was, of course, less than that of the VI-80; the relative harmonic means closely predicted the birds' proportions of choices. This is important to our present concerns because, as noted above, foraging models have predicated their "expected values" upon arithmetic rather than harmonic averaging.

Several other experiments have examined choices between fixed and variable schedules, using bivalued schedules (known technically as "mixed schedules") as the variable alternative. Thus, Fantino (1967) compared a variety of mixed- and fixed-ratio schedules, and Davison (1969) examined mixed- and fixed-interval schedules. Both authors found that the smaller of the two values (ratio or interval) included in a mixed schedule had a disproportionate effect on preferences for that schedule. Fantino found the geometric mean to be a better predictor of his subjects' preferences, and Davison suggested that the effect of short intervals was even greater than either geometric or harmonic averaging would predict. Duncan and Fantino (1970) compared their data on choices between FI and FR schedules with those by Davison, who used mixed FI schedules, and found that neither the range nor the number of different interreinforcement intervals was a critical determinant of choice between two schedules. They suggested that the smaller interreinforcement intervals within a schedule play a predominant role in determining the relative preferences for those schedules. It should be noted that Pubols (1962) also demonstrated that a bivalued distribution of reinforcement delays was preferred over a constant delay with equal mean value when evaluated via rats' choices of alleys in a Y-maze.

The ensuing years have seen extensive study of animals' choices between reinforcement schedules and, thus, of preferences regarding different variable patterns of food delivery. On the one hand, there have been attempts to capture the moment-to-moment dynamics of behavior as it tracks environmental events, and on the other hand, there have been empirical and conceptual developments focusing on larger scale functional relations between choice measures and the average rates of reinforcement provided by the variable schedules that are chosen. However, this work has seldom focused on the distributional characteristics per se of the preferred or nonpreferred schedules. There have been discussions regarding which averaging procedures and transformations appropriately represent sets of data (e.g., Heth, Pierce, Belke, \& Hensch, 1989), but the major emphasis in this literature has been upon the sampling distributions of measures of central tendency-typically the arithmetic means that represent the differing rates of reinforcement provided by the various schedules being compared-rather than upon the underlying distributions of events that those measures of central tendency represent.

The present experiments return to an examination of preferences for bivalued distributions, partly because of their specific relevance to published experiments that have addressed foraging theory and partly to take advantage of their simplicity for manipulating particular distributional characteristics. As in the work of Fantino (1967), pigeons were given choices between FR and bivalued ratio schedules. However, whereas Fantino (1967) assessed preferences through the graded measures of relative response rates on initial VI links of concurrent-chain schedules, the present experiments arranged for quick choices among the ratio schedules-specifically, FI 3-sec initiallink schedules (FI-3) in which only 3 sec must elapse before a response will produce the terminal-link schedule. This achieves greater comparability with research in behavioral ecology (e.g., Caraco et al., 1980). It also yields stronger measures of preference and provides for less interaction between the choice phase and the outcome phase of the procedure (Fantino, 1969).

\section{EXPERIMENT 1A}

\section{Method}

Subjects. The subjects were 6 male White Carneau pigeons (designated as R-6, and I-11 through I-15). They previously had been exposed to several procedures involving concurrent choices between FR and VR schedules of reinforcement. The birds were individually housed in stainless steel cages on a 12:12-h light:dark cycle. The subjects were maintained at $80 \%$ of their free-feeding weights and had free access to water and grit except when in the experimental chamber during experimental sessions.

Apparatus. The experiment was run in three identical standard operant conditioning chambers (Loveland/Gerbrands) for pigeons. Each chamber was equipped with a food hopper and houselights. There also were three keys on the back wall of each chamber, each $22 \mathrm{~cm}$ above the floor. The center key was dark and inoperative. The two side keys were illuminated either yellow or red by $28-\mathrm{V}$ dc bulbs when operative. Reinforcement consisted of 2.75 -sec access to mixed grain in a standard food hopper located directly under the center key. Hopper access was accompanied by the illumination of a 28-V dc bulb inside the hopper housing unit. Procedural events were controlled by a Walter/Palya digital controller (Walter \& Palya, 1984), which was interfaced with an IBM-compatible PC (Pevey, 1988). The controlling software was a version of real-time BASIC (Walter \& Palya, 1985). 
Procedure. Throughout the experiment, choices between FR and bivalued VR schedules (technically, a mixed schedule; see Ferster \& Skinner, 1957) were repeatedly made available to the subjects. In the bivalued VR schedule, the two values ( 1 and 120) had equal probabilities of occurrence. The choice opportunities were arranged by means of a concurrent-chains procedure, which effectively divided each session into discrete trials. Each trial began with the initial link of the chain, denoted by one key being illuminated red and the other yellow, with their positions randomized prior to each initial link. After $3 \mathrm{sec}$ had elapsed (FI-3), the next response on either key produced access to either the bivalued schedule (correlated with the red light) or a fixed schedule (correlated with the yellow light). The fixed schedule was FR-15, FR-30, or FR-60, in separate conditions of the experiment. Once selected, a schedule remained operative and its key remained lighted, while the nonchosen key was made dark and inoperative until a reinforcer was produced. A new trial began immediately after reinforcement. Sessions were terminated after 40 trials or after $60 \mathrm{~min}$ had elapsed, whichever occurred first (nearly always the former).

The subjects were randomly assigned to a sequence of exposure to the FR schedules (see Table 1). Each subject was run in an individual-choice condition for 15 sessions, and sessions were run 5 days per week. To recapitulate: the fixed schedule (FR-15, FR30 , or FR-60) was one of two alternatives available as a consequence of responding in the initial link. The other alternative was always a bivalued VR schedule with an arithmetic mean of 60.5 .

\section{Results and Discussion}

Figure 1 indicates the individual subjects' choices between the various FR schedules versus the bivalued VR schedule over blocks of three successive sessions. Each point in each graph indicates choices of the fixed terminallink schedule, computed as the mean number of choices per 40-trial session. All subjects strongly preferred the bivalued VR in all three choice conditions. Over the last two blocks of sessions, FR terminal-link responding across subjects was below $12 \%$ for the FR- 15 condition, below $6 \%$ for the FR-30 condition, and below $3 \%$ for the FR-60 condition.

Overall, the 6 subjects displayed no substantial preference for any of the FR alternatives. In other words, they displayed insensitivity to the arithmetic mean of the variable schedule relative to each of the fixed schedules, two of which were one half and one fourth the mean value of the VR, respectively. Given that the VR schedule contained only two component values, one can infer that the subjects were extremely sensitive to the minimum value of the VR schedule, thus resulting in their nearly exclusive preference for this schedule. However, these results also allow for the possibility that this apparent attractiveness of the variable schedule over each of the three FRs

Table 1

Order of Exposure in Experiments $1 \mathrm{~A}$ and $1 \mathrm{~B}$ to the FR Schedules in the Concurrent-Choice Versus Bivalued VR

\begin{tabular}{cccc}
\hline Subject & Block 1 & Block 2 & Block 3 \\
\hline R-6 & FR-15 & FR-30 & FR-60 \\
I-11 & FR-30 & FR-60 & FR-15 \\
I-12 & FR-60 & FR-15 & FR-30 \\
I-13 & FR-15 & FR-30 & FR-60 \\
I-14 & FR-30 & FR-60 & FR-15 \\
I-15 & FR-60 & FR-15 & FR-30 \\
\hline
\end{tabular}

could have been at least partly an artifact of insufficient exposure to the FR alternatives. Countering this, we had found in other experiments using similar procedures that pigeons were sensitive to schedule changes even if they sampled one of the two alternatives only occasionally. Nevertheless, in most of the present conditions, the subjects received only scanty exposure to the FR schedules, and the apparent insensitivity might be due to inadequate exposure to the FR schedules. To correct for this possible source of insensitivity, the next experiment arranged for forced exposures to the two available schedules at the beginning of each session.

\section{EXPERIMENT 1B}

\section{Method}

Subjects and Apparatus. The subjects and apparatus were the same as before.

Procedure. Except for one change, the procedure was the same as before, arranging for choices between each FR schedule and the bivalued VR schedule. In that one change, four forced-choice trials immediately prior to each experimental session gave the subjects exposures to the alternative schedules that would be available in that session. The order in which the forced exposures occurred was randomly determined and included two exposures to the current FR and two exposures to the bivalued VR schedule. The latter two exposures included each of the two component schedule values, 1 or 120 , with their sequential order also decided by chance. This arrangement ensured that the subjects encountered each set of alternatives throughout each condition as well as when conditions were changed. For each bird, the sequence of conditions was the same as in Experiment 1A (see Table 1).

\section{Results and Discussion}

Figure 2 shows each bird's choices of the FR schedule over blocks of three sessions. Again, all subjects strongly preferred the bivalued VR to the FR schedule in each choice condition. Over the last two blocks of sessions, FR terminal-link responding across subjects was below $6.5 \%$ for the FR-15 and FR-30 conditions and just above $10 \%$ for the FR-60 condition (due primarily to the two blocks for R-6).

In summary, none of the birds' preferences were well described or predicted by the mean values of the alternatives available to them. Furthermore, forced exposures to the FR schedules did not prevent strong preferences for the bivalued VR. The preference for the VR- 60 relative to the FR-60 could be easily predicted from findings reported by Fantino (1967), which indicated that when the arithmetic mean of a mixed-ratio schedule is equal to that of a fixed ratio, the mixed ratio will be preferred. (Indeed, this could be predicted from a variety of findings reported in the operant literature on choice between fixed versus variable schedules.) Fantino also found that as the mean of the concurrently available fixed alternative was reduced, his birds' preferences for that FR increased. Hence, the present findings for the FR-30 and FR-15 conditions, in which the bivalued VR-60 was still preferred, are not readily predictable from Fantino's results. The discrepancy is probably due to the methodological differ- 

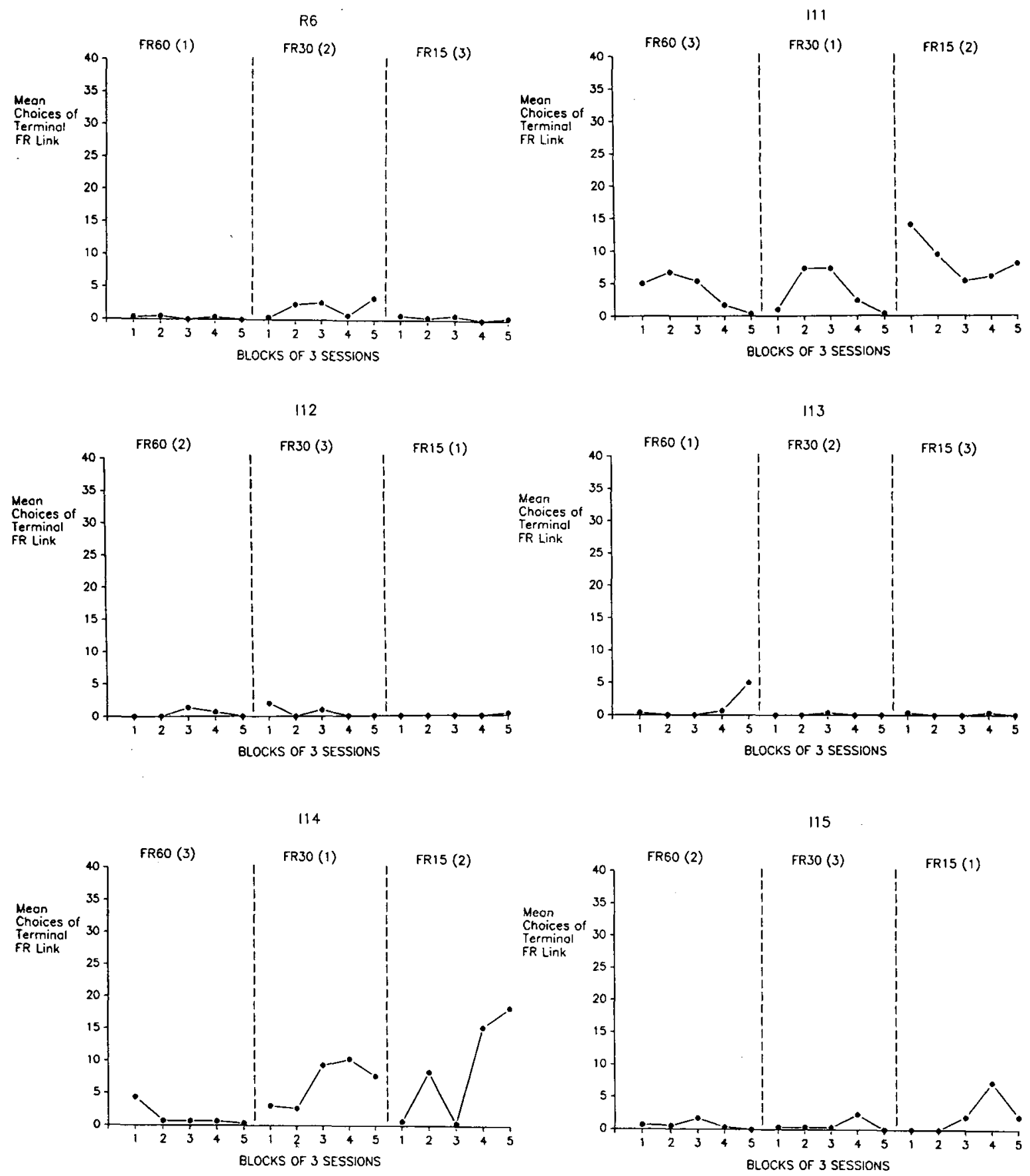

Figure 1. Schedule preferences of each of the 6 pigeons in Experiment 1A, which entailed choices between a bivalued VR and three FR schedules in separate experimental phases. The bivalued schedule always had component values of 1 and 120; the FR values of 15,30 , and 60 were presented for 15 sessions each, their sequence indicated in parentheses. Each point represents the mean number of FR choices per 40-trial session, averaged over blocks of three successive sessions.

ences between the two procedures. As we noted earlier, Fantino used concurrent VI schedules in his initial-link choice phases, and preference was measured in terms of relative rates of responding on those initial links. Our preference measure uses the selection of the schedule itself as an indicator of preference, rather than using a graded indicator of preference. These issues will be considered later in the General Discussion.

While differing somewhat from Fantino's (1967) data, our results do not contradict the geometric averaging that he suggested in interpreting his data. The geometric mean of the bivalued 1 and 120 schedule is 11 . Thus, our FR- 
R6

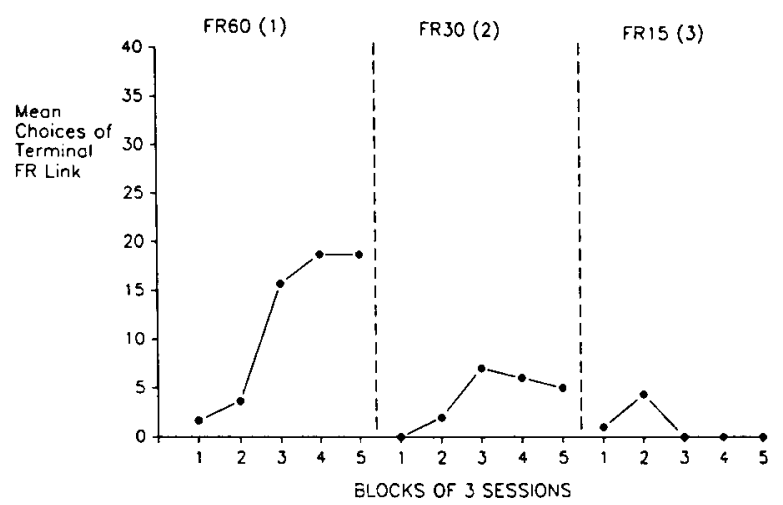

112

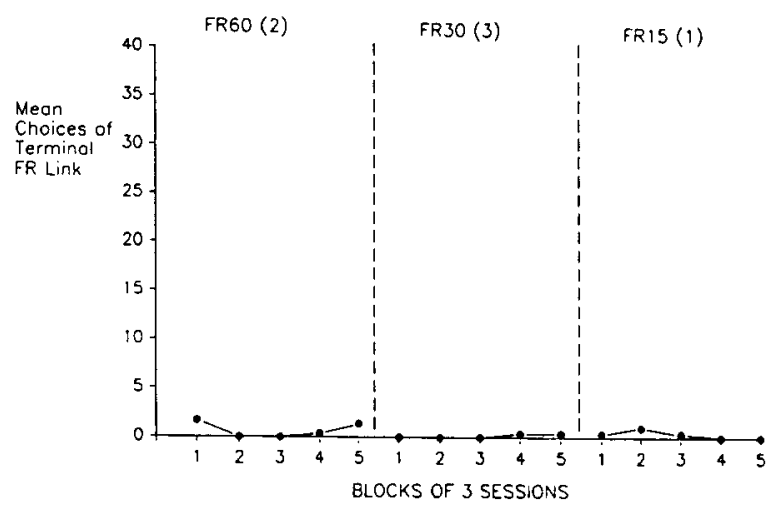

114

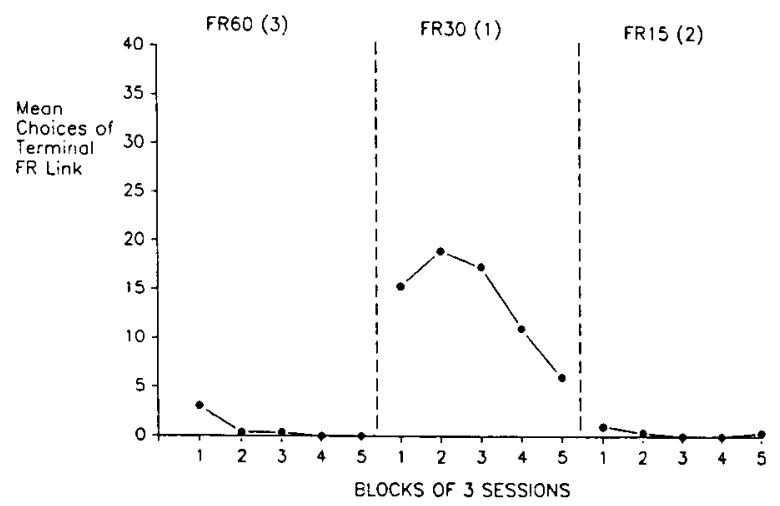

111

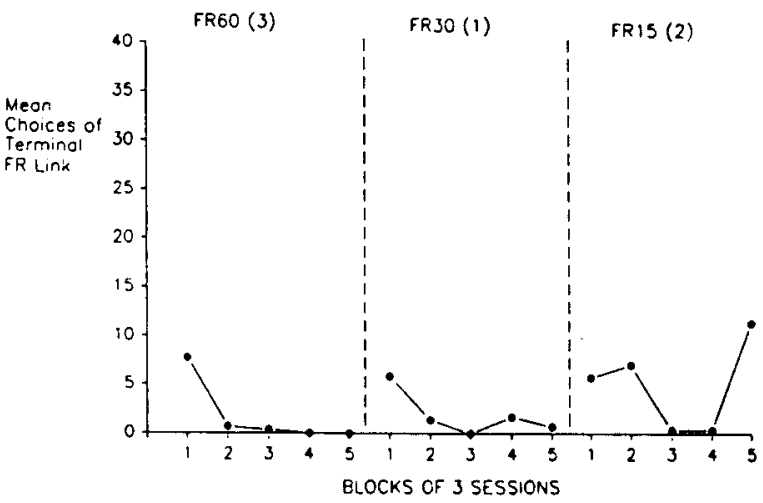

113

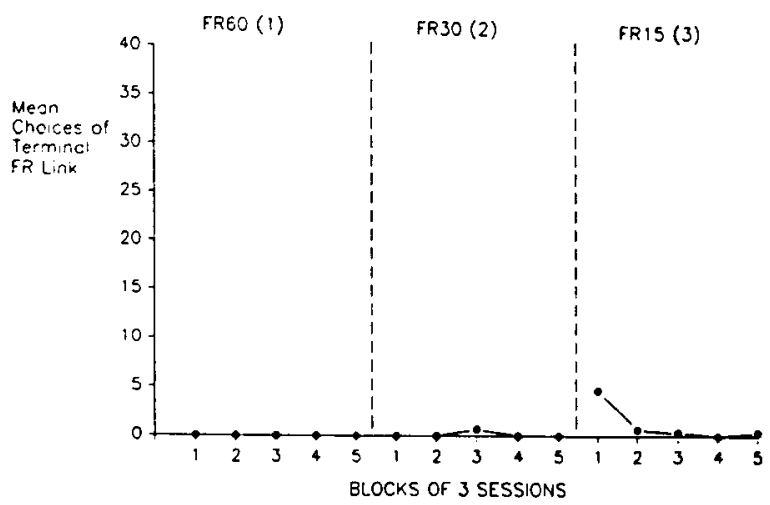

115

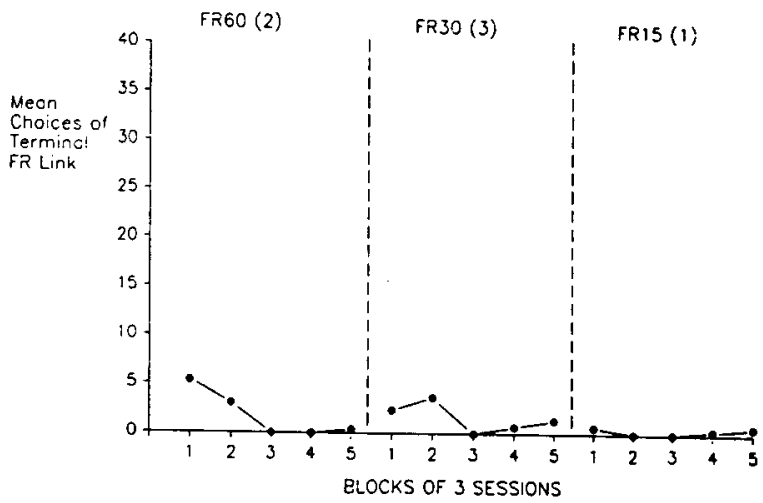

Figure 2. Schedule preferences of each of the 6 birds in Experiment 1B, in which each session of 40 choices was immediately preceded by four forced exposures to the alternatives (FR or VR as in Experiment 1A) that would be available in that session. As in Figure 1, each point represents the mean number of choices per session, averaged over blocks of three consecutive sessions, over the 15 sessions of exposure to each FR condition.

15 was still larger than the geometric mean of our bivalued schedule. Interestingly, when Fantino computed obtained rates of reinforcement on the two schedules, those rates were lowest on the 1 and 99 bivalued schedule, apparently due to the extensive pausing that occurred on the occasions when the mixed schedule was chosen and the first response was not reinforced. This, of course, was the schedule that was most preferred over the FR-50. However, Fantino's assertions regarding geometric averaging are predicated upon rates of reinforcement, not upon the numbers of responses required by the respective schedules. Our present use of an FI-3 initial link may give less 
salience to temporal distributions of events within the procedure, allowing response cost to more directly influence preference between the respective alternatives.

In any case, it would appear that in these conditions the smaller of the VR values (a ratio of 1) pulled the preference toward the bivalued VR even though it was the alternative with the higher mean value. Hence, in the next experiment, the minimum value of the bivalued VR was increased while the corresponding maximum value was simultaneously decreased, keeping the mean value of the bivalued VR at 60 .

\section{EXPERIMENT 2}

\section{Method}

Subjects and Apparatus. The subjects and apparatus were the same as in the previous experiment.

Procedure. The procedure in this experiment also involved choices between an FR schedule $(15,30$, or 60$)$ and a bivalued VR schedule, but the latter involved new component ratios, 15 and 105 , the smaller of which equaled the smallest FR. The larger value was selected so as to keep the arithmetic mean of the VR at 60 . As in Experiment 1B, the subjects were again given 4 presession trials of forced exposure to each of the alternatives that would be available during a given session. Each bird was randomly assigned to one of three sequences of exposure to the FR schedules (see Table 2). In addition to receiving one phase of exposure to each FR, each bird was also returned to its initial condition to provide a within-subject replication. As before, individual sessions consisted of $\mathbf{4 0}$ trials, and each subject was run in a given combination of alternatives for 15 sessions. Sessions were run 5 days per week.

\section{Results}

Figure 3 shows the mean number of FR choices (the number following each FR heading shows the order in which the given condition occurred) for each subject over blocks of three consecutive sessions. For each subject, the number of FR choices increased as the size of the FR decreased. The bivalued VR was clearly preferred when the alternative was the FR-60. The subjects demonstrated indifference or slight preference for the VR when the alternative was the FR-30, despite the fact that the arithmetic mean value of the bivalued VR was twice that of the FR. All subjects clearly preferred the FR during the FR-15 condition, with some of the birds exhibiting exclusive preference for the FR. Nevertheless, there were still several subjects that made a substantial number of VR choices even though the arithmetic mean of this FR was one fourth the value of the arithmetic mean of the

Table 2

Order of Exposure in Experiment 2 to the FR Schedules in the Concurrent-Choice Versus Bivalued VR

\begin{tabular}{ccccc}
\hline Subject & Block 1 & Block 2 & Block 3 & Block 4 \\
\hline R-6 & FR-30 & FR-60 & FR-15 & FR-30 \\
I-11 & FR-60 & FR-15 & FR-30 & FR-60 \\
I-12 & FR-15 & FR-30 & FR-60 & FR-15 \\
I-13 & FR-30 & FR-60 & FR-15 & FR-30 \\
I-14 & FR-60 & FR-15 & FR-30 & FR-60 \\
I-15 & FR-15 & FR-30 & FR-60 & FR-15 \\
\hline
\end{tabular}

VR. Over the last two blocks of sessions, FR terminallink responding across subjects was below $10 \%$ for the FR-60 condition, below $45 \%$ for the FR-30 condition, and above $85 \%$ for the FR-15 condition.

Systematic within-condition trends support the validity of the summarized results. During the course of their exposures to the FR-15 condition, all birds showed increasing tendencies to choose the FR during consecutive sessions of exposure to that condition. In the FR-60 condition, most subjects showed a brief carryover from previous conditions, but after the first one or two threesession blocks of exposure there were few FR choices. Intermediate preferences were consistently observed during the FR-30 condition. That is, the birds were indifferent or slightly preferred the VR. In each bird's return to its initial condition, the initial three-session block showed a carryover from its preceding condition, but preferences systematically changed, reaching values that were not appreciably different from the choices in the final sessions of original exposure to the same condition.

\section{GENERAL DISCUSSION}

In both Experiments $1 \mathrm{~A}$ and $1 \mathrm{~B}$, the pigeons showed very strong and uniform preferences for a bivalued VR schedule even when given forced exposure to the alternate FR schedules at the beginning of each session. The results of Experiment 2 differed systematically from those of Experiments $1 \mathrm{~A}$ and $\mathrm{BB}$ by showing changes in preference as a function of FR size. This result was most notable for the FR-15 condition, where the observed preferences shifted to nearly exclusive preference for that FR. The differing results are presumably attributable to the change of the values of the bivalued VR from 1 and 120 in Experiments $1 \mathrm{~A}$ and $1 \mathrm{~B}$ to 15 and 105 in Experiment 2. It is tempting to identify the change in the lower valued component of the VR (from 1 to 15 ) as the operative variable. However, it must be acknowledged that the range of the bivalued schedule had also been changed, so further experimentation will be needed to assess whether the range, as well as its minimum value, affects the birds' choices. What is clear, however, is that the effective components of bivalued VR schedules are not well expressed in terms of arithmetic averaging.

Our procedure used concurrent FI-3 schedules in the initial-link choice phases for assessing preferences between the fixed and bivalued schedules. In contrast, Fantino (1967) used concurrent VI schedules for the initial links in making somewhat similar comparisons. Concurrent-chains procedures came into general favor within the tradition of free-operant choice studies as an alternative to simple concurrent schedules for measuring schedule preference. This was an acknowledgement that rate of responding on a given schedule will not necessarily represent the organism's preference for that schedule. The initial-link schedules of a concurrent chain provide a measure of preference for a given schedule, distinct from the performance on that schedule, which is confined to 
R6

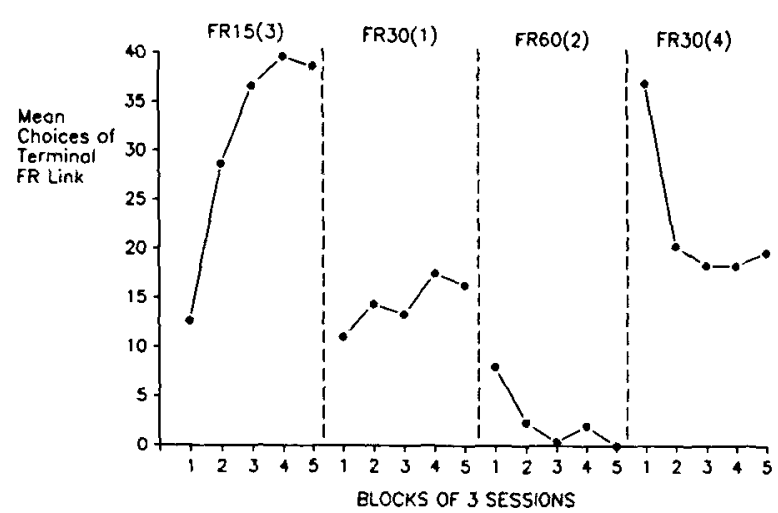

111

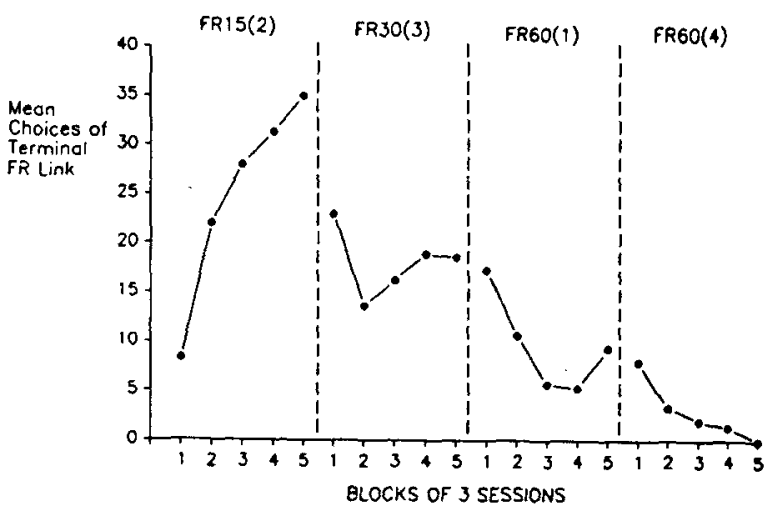

112

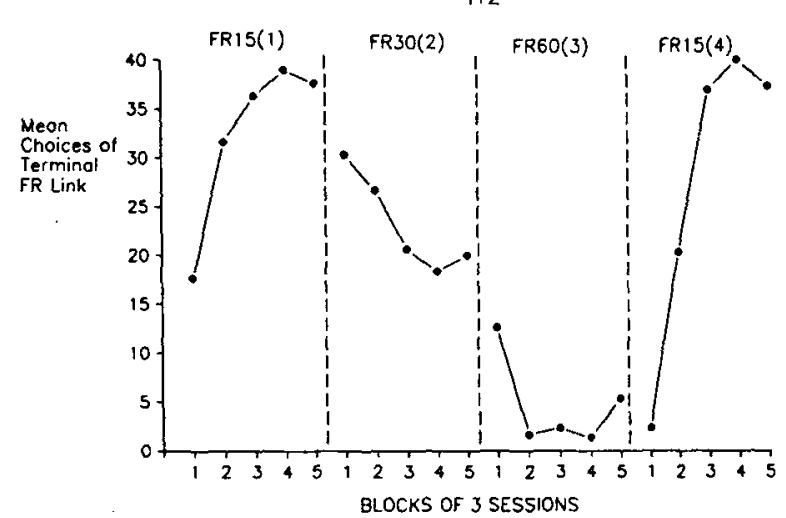

113

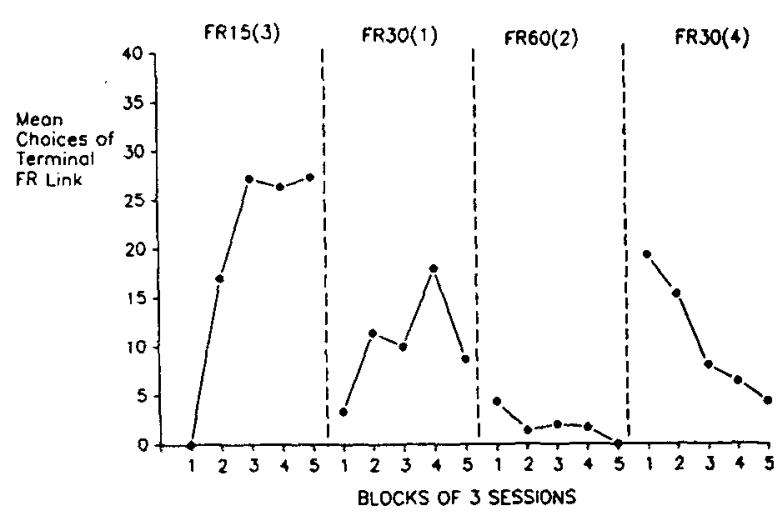

114

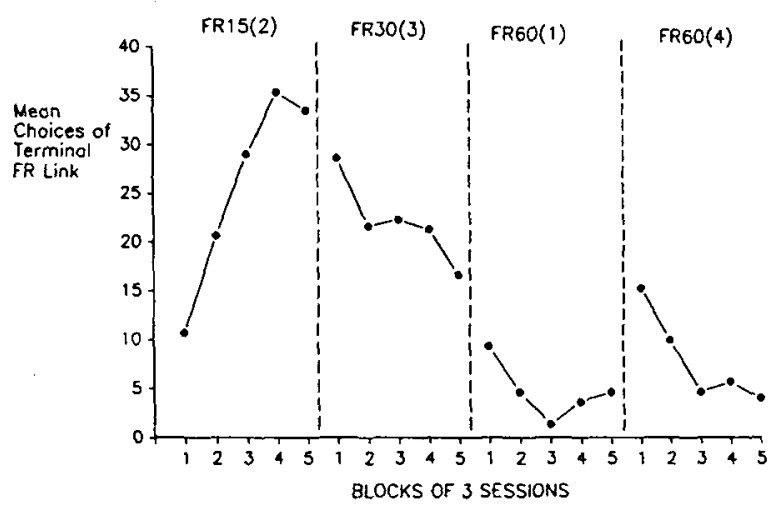

115

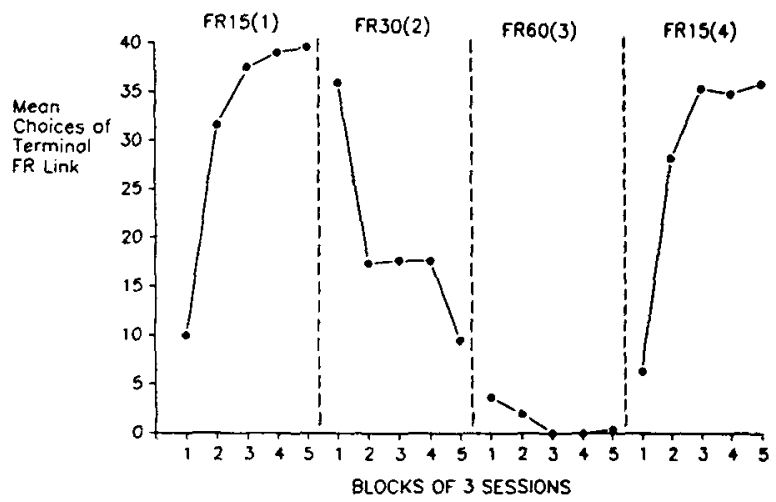

Figure 3. Fixed-ratio choices of each subject in Experiment 2, where the alternative bivalued VR schedule values were 15 and 105. Each session began with four forced exposures to the alternatives that would be available in the subsequent 40 choice trials. Each point shows a mean computed over three consecutive sessions. The FR values (and the order in which they occurred) is indicated at the top of each graph; for each bird, the fourth condition was a replication of its initial FR condition. 
the second link. (Similarly, the behavior of an animal while it is confined to a patch may not indicate the animal's preference for that patch.) Variable-interval schedules came to be favored for the initial-link components for several reasons: they provide a graded measure of preference; substantial changes of preference can occur without appreciably affecting the rate at which access to the preferred schedule is obtained; and scheduled concurrently, the VI contingencies (as distinct from more direct measures of choice) ensure approximately equal numbers of exposures to both alternatives. Like much of the early work with concurrent-chains procedures, Fantino's experiment used identical VI schedules on both keys (either VI-3-min or VI-1.5-min, in different phases of the experiment).

However, while the initial-link VI schedules do have the advantages just noted, they do not provide entirely neutral assessments of preference because characteristics of the choice phase can interact with the chosen alternatives (e.g., Fantino, 1969). Observation of this fact led to the delay-reduction hypothesis, a formulation predicting that the greater the amount of time spent in the choice phase, the weaker will be the measured preference for the favored terminal-link schedule (e.g., see Fantino \& Abarca, 1985). The FI-3 initial-link schedules used in our experiments were chosen to be sufficiently brief that their durations would not become appreciable time components of the overall procedure, yet long enough to minimize extraneous contributions to the measured preference for the terminal-link schedule-for example, pecks elicited to either key simply by trial onset (as might occur if FR-1 schedules were used in the initial links). With these short initial links, the measured preferences should be stronger than are observed with concurrent VIs and should be readily apparent in the total numbers of selections of the respective alternatives, as was observed. In addition, our procedural features heed the suggestion by Wanchisen, Tatham, and Mooney (1989) that temporal contingencies are not as salient to pigeons. Consequently, response requirements were used, since they are presumably more significant to these animals (rats, on the other hand, tend to be more sensitive to temporal contingencies).

The present results suggest geometric averaging as a better portrayal of our birds' preferences than arithmetic averaging, although some of the data indicated that even this may not fully capture observed preferences. The geometric mean of the bivalued 1 and 120 schedule used in Experiments $1 \mathrm{~A}$ and $1 \mathrm{~B}$ is 11 , which is less than the smallest FR that we used, and our subjects strongly preferred the bivalued VR to that FR-15 schedule. On the other hand, the geometric mean of the 15 and 105 schedule is 39.6 , which corresponds fairly well to the birds' preferences in all but the FR-30 condition of Experiment 2: Geometric averaging would predict that the FR30 would be preferred relative to the bivalued VR with a geometric mean of 39.6 , and this clearly was not the case. Definitive evaluation of geometric averaging with respect to these schedule preferences will require more extensive parametric experimentation.
Our finding of increased, if not virtually exclusive, preference for the FR-15 with the VR of 15 and 105 also suggests that the range may be an operative determinant of preference for a schedule. At the same time, it is possible that the minimum value $(1$ or 15$)$ had a disproportionate effect on our subjects' preferences (especially in Experiments $1 \mathrm{~A}$ and 1B). This would be consistent with Fantino's (1967) and Davison's (1969) findings, but this also needs to be evaluated through parametric experiments. Nevertheless, as they stand, the present results have clear implications for contemporary theory, especially the theories that have addressed optimal foraging.

The ecological validity of optimal foraging theories hinges partly upon the fit between the distributions upon which the theories are predicated and the distributions that occur in nature. That means the organism must not only be sensitive to the distributional characteristics that distinguish the experimental alternatives, but also sensitive in the way that the theory assumes or describes. In the case of bivalued distributions used in experimental simulations of foraging, theory has not been sensitive to the determinants of subjects' patterns of choice. Although it surely has characteristics of stochasticity, a bivalued distribution of food items cannot be treated as a uniform distribution that is to be represented by the usual "expected values" of models predicated upon linear integration of probabilities. Thus, while accounts of foraging (as well as choice theories more generally) bring impressive mathematical power and subtlety to the identification of strategies by which individuals maximize their gains, the related experimentation, when it has employed bivalued distributions, lacks ecological validity.

It should be underscored that in the case of bivalued distributions, the mean is theoretical in more than the usual sense, for the subject does not gain exposure to anything even approximating it. Thus, it is a poor characterization of the distribution, and this a priori consideration is amply supported by experimental data. Of course, our findings do not entirely rule out other measures of central tendency as bases for comparing preferences for two sets of variably distributed work requirements; indeed, geometric means are consistent with some of the results. However, the range, minimum value, and other characteristics, such as the effects of systematically manipulated intermediate members of trivalued distributions, need to be further evaluated to make this assessment.

\section{REFERENCES}

Caraco, T., Martindale, S., \& Wittam, T. S. (1980). An empirical demonstration of risk-sensitive foraging preferences. Animal Behaviour, 28, 820-830.

DAvison, M. C. (1969). Preference for mixed-interval versus fixedinterval schedules. Joumal of the Experimental Analysis of Behavior, 12, 247-252.

Duncan, B., Fantino, E. (1970). Choice for periodic schedules of reinforcement. Journal of the Experimental Analysis of Behavior, 14, 73-86.

Fantino, E. (1967). Preference for mixed-versus fixed-ratio schedules. Joumal of the Experimental Analysis of Behavior, 10, 35-43. 
FAntino, E. (1969). Choice and rate of reinforcement. Journal of the Experimental Analysis of Behavior, 12, 723-730.

FAntino, E., AbArCA, N. (1985). Choice, optimal foraging, and the delay-reduction hypothesis. Behavioral \& Brain Sciences, 8, 315-330.

FERSTER, C. B., SxinNer, B. F. (1957). Schedules of reinforcement. Englewood Cliffs, NJ: Prentice-Hall.

Heth, C. D., Pierce, W. D., Belke, T. W., Hensch, S. A. (1989). The effect of logarithmic transformation on estimating the parameters of the generalized matching law. Joumal of the Experimental Analysis of Behavior, 52, 65-76.

KILLEEN, P. (1968). On the measurement of reinforcement frequency in the study of preference. Journal of the Experimental Analysis of Behavior, 11, 263-269.

Leventhal, A. M., Morrell, R. F., Morgan, E. F., Jr., \& PerKINS, C. C., JR. (1959). The relation between mean reward and mean reinforcement. Journal of Experimental Psychology, 57, 284-287.

MAzUR, J. E. (1988). Choice between small certain and large uncertain reinforcers. Animal Learning \& Behavior, 16, 199-205.

Pevey, M. E. (1988). Using an IBM PC to network Walter/Palya experiment controllers. Behavior Research Methods, Instruments, \& Computers, 20, 100-103.

Pubols, B. H., JR. (1962). Constant versus variable delay of reinforcement. Joumal of Companative \& Physiological Psychology, 55, 52-56.

Walter, D. E., PAlYA, W. L. (1984). An inexpensive experimental controller for stand-alone applications or distributed processing networks. Behavior Research Methods, Instruments, \& Computers, 16, 125-134.

WAlter, D. E., PAlya, W. L. (1985). Document s̀et for experiment controller. Unpublished manuscript, Jacksonville State University, Department of Psychology, Jacksonville, AL.

Wanchisen, B. A., Tatham, T. A., Mooney, S. E. (1989). Variable-ratio conditioning history produces high- and low-rate fixedinterval performance in rats. Joumal of the Experimental Analysis of Behavior, 52, 167-179.

(Manuscript received December 3, 1991; revision accepted for publication April 13, 1992.) 